\title{
O "novo" consenso macroeconômico e alguns insights da crítica heterodoxa ${ }^{1}$
}

\author{
Anderson Mutter Teixeira ${ }^{2}$ \\ Fabrício José Missio ${ }^{3}$
}

\section{Resumo}

O objetivo deste trabalho é fazer um survey dos principais papers e argumentos que compõem o chamado "novo" consenso macroeconômico, a fim de elucidar o que alguns autores apresentam como uma nova etapa da História do Pensamento Econômico. Nesse caso, destacam-se os principais esforços na busca pela convergência entre as distintas teorias que suportam essa abordagem, bem como os principais autores que defendem ser essa convergência não só possível, mas desejável. Posteriormente, são sumarizados alguns insights da crítica heterodoxa, em especial, as de cunho póskeynesiano. Sendo assim, o trabalho amplia a discussão proposta, nesta revista, por Arestis e Sawyer (2008). Observa-se ainda que grande parte das críticas feitas a este novo consenso são as mesmas que permeiam o debate entre Keynes e os (neo) clássicos em meados da primeira metade do século XX. Por fim, as conclusões apontam no sentido de mostrar a necessidade de consolidar um construto teórico mais bem articulado para ambas as abordagens.

Palavras-chave: Consenso macroeconômico; Crítica heterodoxa; Política econômica.

\section{Abstract \\ The new consensus macroeconomics and some insights from a heterodox critique}

This paper aims to survey the main contributions to the literature on the "new" consensus in macroeconomics in which the efforts for convergence among the distinct theories that support this approach are discussed. It also seemed appropriate to underscore the main authors who postulate such convergence. The resulting core for economic policies is then presented. Next, some insights on the heterodox critique are summarized. Emphasis was given to the post-Keynesian perspective. Thus, this paper amplifies the debate Arestis and Sawer (2008) proposed in an earlier issue of this journal. At this stage, the reader might find it clear that the critiques are the same as those which governed the debate among Keynes and the classics in the first half of the twentieth century. Conclusions indicate that more research is necessary in this area and that, at both sides of the debate, it is important to summarize the arguments in a coherent fashion in order to consolidate a theoretical framework.

Keywords: Consensus in macroeconomics; Heterodox critique; Economic policy. JEL B16, E32, E42.

(1) Trabalho recebido em 2 de março de 2009 e aprovado em 13 de junho de 2010.

(2) Professor Assistente de Economia da FACE-UFG (Faculdade de Administração, Ciências Contábeis e Ciências Econômicas da Universidade Federal de Goiás), Goiânia, GO, Brasil. Doutorando em Economia pela Universidade de Brasília (UnB) e Bolsista do CNPq (Conselho Nacional de Desenvolvimento Científico e Tecnológico). E-mail: mutterteixeira@yahoo.com.br.

(3) Professor da UEMS (Universidade Estadual de Mato Grosso do Sul), Ponta Porã, MS, Brasil Doutorando em Economia do Cedeplar (Centro de Desenvolvimento e Planejamento Regional) da Universidade Federal de Minas Gerais (UFMG), E-mail: fabriciomissio@gmail.com.

Economia e Sociedade, Campinas, v. 20, n. 2 (42), p. 273-297, ago. 2011. 


\section{Introdução}

Ao longo do século vinte, a economia foi palco de intensas revoluções e contrarrevoluções teóricas que mudaram a compreensão sobre o seu funcionamento. Destaca-se, nesse contexto, a cristalização da macroeconomia como disciplina da ciência econômica cujo objetivo primordial recai, invariavelmente, no entendimento sobre as causas das flutuações econômicas. Atualmente, além das novas abordagens que emergem para a explicação dessas flutuações, evidencia-se, também, o novo conjunto de técnicas e teorias que justificam a implementação e a administração (crível) dos instrumentos de política macroeconômica, sobretudo a partir da definição de novos "papéis" a serem perseguidos pelos (distintos) policymakers.

Entretanto, a consolidação da macroeconomia ao longo do século XX não significou a convergência de argumentos, como fica claro nos debates entre Keynes e os "(neo) clássicos" nos anos trinta, entre Keynesianos e Monetaristas entre os anos sessenta e setenta e/ou entre as distintas escolas que se consolidam principalmente a partir das duas últimas décadas do século vinte. Vale destacar que, além do debate teórico, estabeleceu-se, ao longo desse período, uma clara divergência entre o que, de fato, era discutido na academia e o que era implementado e perseguido pelos policymakers.

Nesse contexto, fica evidente a impossibilidade de convergência ou a falta de comunicação entre tais correntes de pensamento no que tange à possibilidade de apresentação de uma resposta conjunta para os principais problemas econômicos. Todavia, existem esforços no sentido de gerar uma síntese entre esses argumentos como, por exemplo, naqueles empreendidos na consolidação da (velha) síntese neoclássica, que culminou na aceitação da teoria clássica como interpretação econômica dominante. Recentemente, diversos autores têm enfatizado a possibilidade de uma "nova síntese neoclássica" a partir da definição de um núcleo comum de pesquisa, sobretudo no que concerne à condução da política monetária. Mais especificamente, alguns autores defendem a emergência de um Novo Consenso Macroeconômico que busca sintetizar as contribuições teóricas de diversas escolas de pensamento, bem como definem um núcleo central de proposições aceitas por um grande grupo de economistas acadêmicos e policymakers e que têm sido utilizadas como "guia" para a condução da política econômica por diversos bancos centrais.

Todavia, a proposta dessa nova síntese tem sido amplamente criticada, por um lado, porque é incapaz de consolidar-se enquanto construto teórico e, por outro, porque ignora desenvolvimentos teóricos e empíricos recentes da literatura heterodoxa. Em outras palavras, critica-se essa nova síntese por ser um conjunto de proposições ancoradas na teoria mainstream e que, portanto, seguem uma 
metodologia e um conjunto de hipóteses que são, juntamente com seus resultados, questionáveis, não só do ponto de vista empírico, mas também da forma como os problemas econômicos são tratados.

Tendo em vista essa discussão, o objetivo deste paper é fazer um survey sobre essa literatura, apresentando as características desse Novo Consenso Macroeconômico pela análise dos principais trabalhados que sustentam essa tese assim como algumas considerações críticas apontadas pela literatura heterodoxa, principalmente aquelas advindas da inspiração pós-keynesiana. Nesse caso, amplia-se a discussão iniciada por Arestis e Sawyer (2008), em artigo publicado nesta revista, em que se busca explorar com maior ênfase as ideias que compõem o core do novo consenso, na medida em que novas críticas lhe são feitas. Além disso, são apresentados alguns elementos da teoria pós-keynesiana que se entende como importantes contribuições desse pensamento e que, obviamente por fundamentarem-se em um núcleo teórico distinto, estão excluídas da abordagem proposta pelo Novo Consenso.

Para cumprir com os objetivos, o presente trabalho encontra-se dividido em cinco seções, além desta introdução e das considerações finais. A seção 1 e a seção 2 apresentam as características e objetivos desse Novo Consenso, bem como uma interpretação acerca de suas origens teóricas. A terceira seção apresenta uma primeira formalização dessa abordagem. A quarta seção é dedicada à apresentação de algumas críticas heterodoxas e, finalmente, a quinta seção apresenta alguns elementos da interpretação de inspiração pós-keynesiana sobre o processo de crescimento em economias capitalistas de produção.

\section{0 novo consenso macroeconômico: objetivos e características}

Em meados dos anos noventa, economistas como Blinder (1997) e Blanchard (1997), entre outros, passaram a defender a existência de um novo consenso macroeconômico, sendo este, tal como explicitado em Taylor (1997, p. 233), um "set of key principles - a core of macroeconomics about which there is wide agreement". Este "core macroeconomics" é na verdade um conjunto de proposições centrais acerca de como deve ser interpretado o processo de crescimento econômico das economias capitalistas. Segundo Taylor $(1997,2000)^{4}$, podem-se destacar cinco elementos centrais:

i) A análise das flutuações no produto real de longo prazo da economia é entendida a partir da teoria neoclássica do crescimento, ou seja, via deslocamentos da função de produção. Em outros termos, considera-se válida a hipótese da decomposição ciclo-tendência.

(4) Blinder (1997) também expressa um núcleo macroeconômico em quatro pontos, semelhantes aos aqui apresentados. 
ii) Não há um trade off entre a taxa de inflação e desemprego no longo prazo. Nesse caso, o consenso é de que uma política monetária expansionista resultará em uma maior taxa de inflação de longo prazo, ao passo que deve manter a taxa de desemprego inalterada. Logo, defende-se que o Banco Central deve adotar uma meta de longo prazo para a inflação e manter-se a ela (Taylor, 1997).

iii) Existe um trade off temporário entre inflação e desemprego. Este resultado, apresentado em trabalhos de Fischer (1977), Taylor (1979) e Calvo (1983), é derivado da hipótese de expectativas racionais com rigidez de preços e salários. Apesar da possibilidade de realizar o trade off de curto prazo, Taylor (1997, p. 234) defende que "monetary policy should keep the growth of aggregate demand stable in order to prevent fluctuations in real output and inflation".

iv) As expectativas dos agentes econômicos são altamente sensíveis às ações de políticas econômicas. Assim, as estimativas de política fiscal e, em especial, da monetária são bem-vindas, ou seja, o desenvolvimento de relatórios sobre o comportamento dos principais agregados macroeconômicos e sobre ações tomadas e a serem tomadas pelos policymakers e o uso de previsão da inflação como meta intermediária são elementos essenciais para complementar o conjunto de informações dos agentes.

v) A demanda agregada é sensível à taxa de juros respondendo, portanto, a variações na política monetária. Diante disso, os policymakers devem realizar mudanças graduais (na política monetária) via regras de conduta ou função de reação, sendo a taxa de juros de curto prazo o instrumento de política monetária que deve ser ajustado em resposta aos choques econômicos.

Segundo Blanchard (1997), o que deve ser destacado nesse novo consenso é a convergência entre a teoria e a prática da política monetária, bem como a interação entre os policymakers e os economistas acadêmicos. Ou ainda, segundo Goodfriend \& King (1997), deve-se enfatizar o fato de, nos últimos anos, a macroeconomia estar se movendo na direção de uma nova Síntese Neoclássica. No espírito da velha síntese, que captava elementos tanto de origem clássica quanto keynesiana, a proposta atual é uma combinação de elementos desenvolvidos pelas correntes Novo Clássico, Novo Keynesiano e Real Business Cycle, que se insere em uma moldura teórica e prática desenvolvida, sobretudo, pelos monetaristas, com ênfase na condução do instrumento monetário.

Assim, nesse contexto de destaque para a política monetária, ressaltam-se as recomendações sobre como deve ser sua conduta num cenário de convergência entre as proposições teóricas. Especificamente sobre estas recomendações, Goodfriend e King (1997, p. 26) sintetizam quais devem ser os objetivos: "Thus the recommendation is that monetary policy should stabilize the path of the price level in order to keep output at its potential. This policy is "activist" in that the 
authority must manage aggregate demand to accommodate any supply - side disturbances to output".

Do ponto de vista prático, a implementação do regime de metas de inflação tem um papel de destaque nesse Novo Consenso, uma vez que cumpre com os objetivos supracitados. Além disso, esse regime se destaca pela credibilidade e pela transparência de objetivos e pela simplicidade no manejo do instrumento de política econômica, a saber, a taxa de juros, cujo objetivo é, entre outros, servir como um guia capaz de coordenar as expectativas inflacionárias dos agentes.

Corroborando a temática de expor algumas características do Novo Consenso Macroeconômico, é importante destacar os trabalhos desenvolvidos por Arestis e Sawyer (2002a; 2002b; 2002c; 2006) em que são apresentadas algumas proposições sobre essa nova estrutura teórica:

i) Propriedade da neutralidade da moeda, cujo valor de equilíbrio das variáveis reais é independente da oferta de moeda, e o processo de mitigação da inflação, realizado pela política monetária, em especial, pela taxa nominal de juros. A curva de Phillips é vertical, ou seja, no longo prazo não há trade off entre inflação e desemprego ou entre inflação e produto. A taxa de desemprego no longo prazo é determinada pelas condições de oferta e pelas condições do mercado de trabalho negligenciando-se, portanto, fatores de demanda. Em outras palavras, no longo prazo, a economia opera na "Non Accelerating Inflation Rate of Unemployment" (NAIRU).

ii) Existe uma âncora nominal, cujo objetivo é determinar o equilíbrio nominal de uma economia. Atualmente a âncora nominal padrão é a meta de inflação.

iii) Credibilidade e transparência são reconhecidas como elementos-chave para o sucesso da âncora nominal, pois tais elementos inibem problemas de inconsistência dinâmica e/ou de viés inflacionário à semelhança de KydlandPrescott (1977) e Barro-Gordon (1983a; 1983b). Como resultado, um banco central independente é bem-vindo para mitigar tais procedimentos e elevar a credibilidade dos agentes econômicos em torno do commitment.

iv) A política monetária via regra de conduta é postulada como o principal instrumento de política econômica. Deste modo, a política fiscal exerce influência secundária no objetivo primordial de estabilidade do nível de preços.

\section{Algumas observações sobre as origens do Novo Consenso}

A consolidação dessa nova estrutura teórica, conforme propõem alguns autores, é fruto de intenso debate entre os economistas no final do século vinte. 
Até o início dos anos noventa, tinha-se a impressão de que a relação entre as correntes macroeconômicas era totalmente estanque. Porém, recentemente, observa-se um esforço na busca pela convergência entre as principais proposições macroeconômicas, principalmente no que se refere à forma como deve ser conduzida a política econômica, em especial, a monetária.

Segundo Goodfriend (2005, p. 250), essa convergência em termos teóricos é a junção de "embody key components from keynesian, monetarist, rational expectations and real business cycle macroeconomics", acrescido da questão prática, fruto, em grande parte, do experimento monetarista desenvolvido na economia americana a partir de 1979. Nesse caso, a desinflação da economia contou com a liderança de Paul Volcker como presidente do Federal Reserve Board. Ou seja, o sucesso do processo de mitigação da espiral inflacionária, via política monetária, sem a necessidade de políticas de controle de preços, salários e ações de âmbito fiscal, indica a consolidação, em termos teóricos e práticos, de um novo arranjo institucional.

Ainda nesse sentido, segundo McCallum (1999), o novo consenso macroeconômico está preocupado em atender a duas proposições teóricas da macroeconomia atual: a Crítica de Lucas e a responsabilidade exclusiva que os bancos centrais devem ter com a estabilidade de preços. Ou seja, a combinação das proposições teóricas em conjunto com as evidências práticas executadas pelos policymakers gera o que o autor denominou de "multicolinearity problem". Nesse contexto, o autor postula que:

Thus we have theory, formal evidence, and informal "experimental" evidence all pointing in the same direction - toward the idea that from a long-term perspective monetary policy's main influence is on growth of the price level with little or no lasting effect on real output's level or growth rate. From this conception it is a natural step to view inflation prevention as the main macroeconomic duty of a modern central bank, with a secondary objective of dampening cyclical fluctuations, and today's general policy climate falls into place (1999, p. 12, grifos adicionados).

Outra iniciativa no intuito de compreender as origens desse novo consenso foi desenvolvida por Zouache (2004), que defende ser esta abordagem uma agenda de pesquisa estendida da corrente Ciclo Reais de Negócios, adicionada de alguns microfundamentos da corrente Novo Keynesiana. De maneira ligeiramente diferente de Woodford (2008), o autor sumariza que o mesmo não é somente fruto dos progressos metodológicos da corrente Ciclo Reais de Negócios, mas, sim, uma combinação com os avanços metodológicos da corrente Novo Clássica.

Em um artigo recente, Beans (2007, p. 171) sintetiza quatro características primordiais do Novo Consenso: i) the primacy of monetary policy for managing demand, ii) the virtues of central bank independence, iii) a focus on ends rather 
than means and iv) the importance of managing expectations. Em relação a essas características, tem-se que a prioridade na administração da demanda agregada é correlacionada com os pressupostos monetaristas, alinhando-se à ideia de que o instrumento de conduta é a taxa de juros, uma vez que esta taxa pode ser modificada agilmente, em detrimento do uso da política fiscal. Em relação à segunda, diante de problemas como o de inconsistência dinâmica e de viés inflacionário, defende-se a necessidade de se desenvolver um banco central comprometido com a estabilidade de preços via delegação dessa tarefa a um "expert" avesso à inflação, em detrimento de possíveis staffs políticos eleitoreiros ou subordinados a governos populistas.

No que tange às expectativas, Bean (2007, p. 173) justifica que "there is widespread agreement that there is no exploitable long run trade-off between activity and inflation, even though one may be present in the short run". Esse argumento está alinhado ao objetivo do Novo Consenso Macroeconômico, expresso em Goodfriend e King (1997) e Goodfriend (2005; 2007), ou seja, que a prioridade da política monetária deve ser a estabilidade de preços.

Por fim, o papel chave das expectativas e da credibilidade é destacável mediante o papel das expectativas racionais na formulação das políticas por parte dos policymakers. Para Mishkin (2007, p. 2), as proposições chaves teóricas desenvolvidas nos últimos anos que sustentam a atual conduta de política monetária podem ser sintetizadas nos seguintes pontos:

1- Inflation is always and everywhere a monetary phenomenon; 2-Price stability has important benefits; 3-There is no long-run tradeoff between unemployment and inflation; 4-Expectations play a crucial role in the determination of inflation and in the transmission of monetary policy to the macroeconomy; 5-Real interest rate need to rise with higher inflation, i.e. The Taylor Principle; 6-Monetary policy is subject to the time inconsistency problem; 7-Central Bank independence helps improve the efficiency of monetary policy; 8-Commitment to a strong nominal anchor is central to producing good monetary outcomes; and, 9-Financial Frictions play an important role in business cycles.

O principio de Taylor (item 5) que melhor representa a conduta atual dos policymakers é a equação desenvolvida inicialmente por Judd e Rudebush (1998), Clarida, Galí e Gertler (1998; 1999; 2000) e/ou como apresentada nos livros-textos de política monetária de Woodford (2003) e Galí (2008), entre outros. Em tais especificações, os autores usaram uma versão modificada da função de reação original de Taylor, sendo que a mesma passou a depender dos valores esperados da inflação e do produto, enfatizando-se, assim, o papel fundamental das expectativas.

Assim, como observado, muitas dessas proposições foram desenvolvidas por economistas da corrente Novo Clássica - como a hipótese de expectativas 
racionais e de agentes otimizadores - ou pela corrente dos Ciclos Reais de Negócios - como os modelos de equilíbrio geral dinâmicos - ou ainda, pela corrente Novo Keynesiana - como nos modelos que incorporam os microfundamentos e/ou as análises mediante a presença de rigidez real e nominal e da concorrência monopolística. Assim, Mishkin (2007, p. 17) postula que:

The real business cycle approach makes use of stochastic general equilibrium growth models with representative optimizing agents. The resulting new class of models, in which new-Keynesian features such as nominal rigidities and monopolistic competition are added to the frictionless real business model, have become known as "dynamic stochastic general equilibrium (DSGE) models ${ }^{5}$.

\section{O Novo Consenso: uma primeira formalização}

Com o objetivo de expressar quantitativamente a moldura teórica que vem sendo utilizada por inúmeros bancos centrais, o novo consenso macroeconômico pode ser formalmente expresso de várias maneiras, como pode ser encontrado nos trabalhos pioneiros de Clarida, Galí e Gertler (1999), McCallum (1999; 2001; 2005), Mankiw (2001), Meyer (2001), Arestis e Sawyer (2002a; 2002b; 2002c; 2006) ou nos modelos para economias abertas, como em Arestis (2007) e Angeriz e Arestis (2007). Entretanto, como destacado por Meyer (2001), em síntese, esse novo consenso pode ser representado a partir de um modelo dinâmico com três equações. Assim, no que se segue, são apresentados alguns trabalhos que utilizam essa formalização destacando que, muito embora sua especificação possa diferir no número de variáveis ou no número de defasagens utilizadas, a essência (dessas equações) permanece a mesma ao longo dos trabalhos.

Essas equações são identificadas como: i) Equação Demanda Agregada, ii) Curva de Phillips e iii) uma regra de política monetária. Em relação à primeira equação, a representação segue a estrutura da antiga curva IS - com a diferença agora de que ela deriva de uma estrutura de otimização intertemporal - que relaciona a forma como o produto responde às mudanças na taxa de juros real. A segunda equação é uma relação de ajustamento de preços que especifica o comportamento da inflação em resposta às variações na capacidade produtiva e nas expectativas e, por fim, a terceira equação é uma regra de política monetária (Meyer, 2001).

O modelo desenvolvido por Clarida, Galí e Gertler (1999) é um dos que fundamentam este novo arranjo monetário. Este modelo se enquadra na

(5) Arestis e Mihailov (2007) argumentam que a recente literatura sobre política monetária vem incorporando os mais recentes progressos da macroeconomia, gerando os microfounded dynamic - stochastic general - equilibrium (DSGE). Tais modelos são denominados, na visão de Clarida, Galí e Gertler (1999), "The science of monetary policy”. Na mesma linha, podem ser caracterizados como Novo Consenso Macroeconômico, como Mayer (2001), pois ambos os trabalhos apresentam, com algumas diferenças, uma Equação IS, uma equação de ajustamento de preços ou uma Nova Curva de Phillips e uma regra a la Taylor. 
perspectiva de que a conduta de política monetária tem papel chave na determinação da atividade econômica de curto prazo, mediante a presença de rigidez temporária dos preços nominais. Diante disso, o modelo está baseado em uma estrutura de equilíbrio geral dinâmico com moeda e uma temporária rigidez nominal dos preços, somado ao comportamento dinâmico dos agentes, que ocorre via processo de otimização das decisões das firmas e dos consumidores.

Formalmente, definindo $\pi_{t}$, a inflação ocorrida no período $t$ e $i_{t}$, a taxa nominal de juros, é possível representar o comportamento da economia a partir de duas equações, uma do lado da demanda, denominada de Curva $\mathrm{IS}^{6}$, e a outra do lado da oferta, Curva de Phillips, sendo;

$$
\begin{aligned}
& x_{t}=-\varphi\left(i_{t}-E_{t} \pi_{t+1}\right)+E_{t} x_{t+1}+g_{t} \\
& \pi_{t}=\lambda x_{t}+\beta E_{t} \pi_{t+1}+u_{t}
\end{aligned}
$$

em que $\pi_{t}$ é a taxa de inflação do período $t$, definida como a mudança percentual no nível de preços entre $t-1$ e $t ; x_{t}$, o hiato do produto; $E_{t} \pi_{t+1}$, a expectativa de inflação em $t$ para o período $t+1 ; E_{t} x_{t+1}$, a expectativa do hiato do produto em $t$ para o período $t+1$; e $R_{t}$, a taxa de juros nominal de curto prazo. Além disso, $g_{t}$ e $u_{t}$ são termos de erro que obedecem, respectivamente, a:

$$
\begin{aligned}
& g_{t}=\mu g_{t-1}+\hat{g}_{t} \\
& u_{t}=\rho u_{t-1}+\hat{u}_{t}
\end{aligned}
$$

em que $0 \leq \mu, \rho \leq 1$ e $\hat{g}_{t} e \hat{\mathrm{u}}_{\mathrm{t}}$ são variáveis randômicas independentes e identicamente distribuídas (i.i.d) com média zero e variâncias $\sigma_{s}^{2}$ e $\sigma_{u}^{2}$, respectivamente.

Cabem aqui algumas considerações sobre essa nova curva IS, uma vez que existe uma dependência no comportamento da demanda agregada com relação às variações nas expectativas esperadas em termos de produto e da taxa de juros. Assim, um aumento esperado no produto irá elevar o produto corrente, pois os

(6) Conforme destacado pelos autores, a equação (2) é obtida à luz da identidade $Y_{t}=C_{t}+G_{t}$ sendo $C_{t}$ e $G_{t}$ o consumo das famílias e o gasto do governo, respectivamente. Logo, pode-se escrever a equação de Euler do consumo em termos lineares em log como: $Y_{t}-e_{t}=-\varphi\left(i_{t}-E_{t} \pi_{t+1}\right)+E_{t}\left(y_{t+1}-e_{t+1}\right)$ em que $e_{t}=-\log \left(1-G_{t} / Y_{t}\right)$ é obtida de forma exógena. Deste modo, usando a definição de hiato de produto e fazendo $g_{t}=\left(\Delta Z_{t+1}-\Delta e_{t+1}\right)$, é possível chegar à equação (2). 
indivíduos irão preferir suavizar o consumo futuro. Já o efeito negativo do aumento da taxa de juros ocorre a partir da substituição intertemporal dos agentes entre consumo e poupança. Ou seja, uma elevação na taxa de juros pode elevar o nível de poupança em detrimento do consumo presente.

Deve-se destacar, ainda, o papel das expectativas nesta equação. Neste caso, leva-se em consideração o impacto das expectativas futuras na atividade econômica corrente. Ou seja, a trajetória do produto agora é determinada não somente pela taxa de juros e pelos choques de demanda corrente, mas, também, pela trajetória futura das expectativas. Deste modo, ciente de que a política monetária afeta a taxa de juros real da economia, via suposição da existência de algum grau de rigidez temporária, Clarida, Galí e Gertler (1999) sumarizam que tanto ações de política corrente como as esperadas têm efeitos sobre a demanda agregada.

A equação (2), que representa a Curva de Phillips, é derivada de um problema explícito de otimização em um contexto de competição monopolística, em que cada firma define seu nível de preços sujeitos à frequência de ajustes futuros. A principal diferença dessa proposição em relação à curva de Phillips original é a inclusão da variável expectacional em relação à inflação futura, $E_{t} \pi_{t+1}$, no lugar da expectativa passada, $E_{t-1} \pi_{t}$. Ainda, com relação a esta equação, observa-se que o coeficiente do hiato do produto $\lambda$ é decrescente em relação ao grau de rigidez dos preços e $u_{t}$ representa os possíveis choques de oferta.

Por fim, é necessário especificar uma regra de conduta baseada no instrumento taxa de juros em detrimento do controle de oferta de moeda. Nesse caso, os autores Clarida, Galí e Gertler (1999) apresentam uma inovação em relação à Regra de Taylor (1993) tradicional, uma vez que agora fica explícito na equação o papel primordial das expectativas inflacionárias.

$$
i_{t}^{*}=\alpha+\gamma_{\pi}\left(E_{t} \pi_{t+1}-\bar{\pi}\right)+\gamma_{x} x_{t}
$$

Como destacado pelos autores, essa regra responde à inflação esperada, ao contrário de se concentrar em estimativas de inflação passada. Deste modo, essa regra de Taylor é consistente com as equações apresentadas anteriormente.

Outra maneira formal de expressar o Novo Consenso Macroeconômico é apresentada por McCallum (1999), resultado dos seminários realizados pelo National Bureau of Economic Research (NBER) e Ribsbank. Uma das características relatadas pelo autor é ausência de qualquer tipo de equação para expressar a demanda por moeda. Mais especificamente, o modelo pode ser representado pelas equações: 


$$
\begin{aligned}
& Y_{t}=\alpha_{0}+\alpha_{1} E_{t} y_{t+1}+\alpha_{2}\left(R_{t}-E_{t} \Delta p_{t+1}\right)+\alpha_{3}\left(g_{t}-E g_{t+1}\right)+V_{t} \\
& \Delta p=E_{t} \Delta p_{t+1}+\alpha_{4}\left(y_{t}-\overline{y_{t}}\right)+U_{t} \\
& R_{t}=\mu_{0}+\mu_{1}\left(\Delta p_{t}-\stackrel{*}{\Delta p}\right)+\mu_{2}\left(y_{t}-\bar{y}_{t}\right)+C_{1}
\end{aligned}
$$

em que $y_{t}$ é o log do produto; $p_{t}$, o log do nível de preços; $R_{t}$, a taxa nominal de juros; $\Delta p_{t}$ representa a inflação; $R_{t}{ }^{-} E_{t} \Delta_{p_{t+1}}$, a taxa de juros real; $y_{t}-\bar{y}_{t}={ }^{*} y$, o produto potencial; $g_{t}$ representa o log dos gastos do governo; $E t$ denota o operador expectacional ou a informação disponível período t e $E_{t} \Delta_{p_{t+1}}$ é a expectativa racional formada no período $t$ de $\Delta p_{t+1}$, a inflação do período futuro. As equações acima representam, respectivamente, a curva IS, a curva de Phillips e uma regra de política monetária semelhante àquela expressa por Taylor (1993).

Por intermédio das três equações listadas acima, McCallum (1999) afirma que existem condições para traçar a trajetória das três variáveis endógenas do modelo: produto ou produto potencial, taxa de juros e nível de preços. Uma característica marcante desse modelo é a ausência de uma equação para determinar a demanda por moeda, refletindo suas duas propriedades: i) a primeira propriedade é que o termo estoque real $m_{t}^{-} p_{t}$ aparece dentro da equação da curva IS e ii) a segunda propriedade está relacionada à hipótese da "separabilidade" obtida via função de utilidade indireta. De forma análoga, isso reflete que os componentes de uma função utilidade podem ser determinados de maneira independente, como, por exemplo, a demanda por bens de consumo e os encaixes reais, entre outros.

Ainda sobre essas equações, o termo $E_{t} Y_{t+1}$, presente na equação que representa a curva IS, reflete o comportamento otimizador e racional dos agentes econômicos e das firmas em torno das decisões de consumo, como observado por Clarida, Galí e Gertler (1999). Com relação à curva de Phillips, o termo $E_{t} \Delta p_{t+1}$,

em vez de $E_{t-1} \Delta p_{t}$, reflete o papel das expectativas racionais, ou seja, a expectativa em relação ao nível de preços esperados sobre as decisões dos preços das firmas.

Finalmente, cabe destacar que, neste modelo, evidencia-se o papel primordial da regra de Taylor como regra padrão utilizada nos principais modelos de conduta de política monetária. Isso é confirmado no argumento de McCallum (2005, p. 288) "undoubtedly represents a move in the direction of realism since 
actual central banks of industrial countries almost invariably use some short-term nominal interest rate as their operating target".

Em Meyer (2001), postula-se a existência de um de novo consenso que pode ser expresso em termos de três equações básicas:

$$
\begin{aligned}
& Y_{t}^{g}=a Y_{t-1}^{g}+b E_{t}\left(Y_{t+1}^{g}\right)-C\left[R_{t}-E_{t}\left(P_{t+1}\right)\right]+X_{t} \\
& p_{t}=d\left(Y_{t}^{g}\right)+w_{1} p_{t-1}+w_{2} E_{t}\left(p_{t+1}\right)+Z_{t}, W_{1}+W_{2}=1 \\
& R_{t}=r^{*}+E_{t}\left(p_{t+1}\right)+f Y_{t-1}^{g}+g\left(p_{t-1}-p^{t}\right)
\end{aligned}
$$

em que (9) é a Equação de Demanda Agregada, (10) Curva de Phillips e (11) uma regra de política monetária, sendo $Y^{g}=g a p$ do produto (em pontos percentuais); $R_{t}=$ taxa de juros nominal; $r^{*}=$ taxa de juros de equilíbrio; $p=$ inflação; $p^{T}=$ igual a meta de inflação; $x$ e $z$ são choques estocásticos e todos os coeficientes são positivos.

Nesse modelo, a equação de demanda agregada é uma versão dinâmica da velha curva IS do modelo IS-LM, no qual o nível de produto ou o gap do produto depende da taxa de juros, sendo que, para o caso atual, a especificação é mais genérica, uma vez que permite captar a influência tanto de elementos defasados do produto como do produto esperado. A curva de Phillips, por sua vez, relaciona o comportamento do nível de preços com o gap do produto da inflação passada e da inflação esperada. Nesse caso, como destacada por Meyer (2001), a inflação passada captura o efeito do papel da rigidez dos preços nominais e a inflação esperada captura o efeito das expectativas quanto ao preço futuro. Por fim, a regra de Taylor é apresentada como instrumento de política monetária. Sua trajetória é determinada pelo desvio do produto em torno do produto potencial e da inflação em relação à meta preestabelecida.

Nessas três equações admite-se a rigidez de preços no curto prazo, o que permite flutuações na atividade econômica neste período, sendo que, no longo prazo, ocorre uma transição estável para o ponto de equilíbrio dado pelo produto potencial. Com isso, busca-se estabilizar os preços e fazer com que a economia cresça em torno do produto potencial. Dentro desta sequência, a incorporação do comportamento forward-looking retrata o papel de destaque das expectativas racionais na conduta dos policymakers.

Por fim, é pertinente destacar o procedimento pelo qual a meta de inflação é almejada no conjunto dessas três equações que compõem o modelo do novo consenso. O processo inicia-se com a determinação da taxa de juros via regra de Taylor (13), em que variações na taxa de juros afetam a demanda agregada que, por sua vez, tem impactos sobre a inflação. Assim, como sintetizado por Arestis e 
Sawyer (2002a, p. 4): "Higher (lower) interest rate tends to reduce (increase) aggregate demand, and lower (higher) aggregate demand is assumed to reduce (increase) the rate of inflation". Os autores denominam esse comportamento (variações na taxa de juros) de ajustamento sistemático ou, como em Arestis (2007), de "symmetric approach to inflation targeting".

Nesse sentido, uma vez feita essa apresentação sumária dos modelos que vêm sendo indicados como integrantes desse novo consenso macroeconômico, a seção seguinte busca tecer algumas considerações críticas sobre sua estrutura teórica. Não obstante, essas considerações limitam-se ao arcabouço keynesiano e pós-keynesiano, ainda que sejam também compartilhadas por outras correntes do pensamento econômico.

\section{A crítica heterodoxa: aspectos gerais}

A crítica heterodoxa aqui explicitada segue a linha de trabalho de Lavoie (2006). Segundo este autor, a partir da estrutura formada pelas três equações que compõem o novo consenso, é possível mostrar que a mudança de uma hipótese nas suas formulações modifica completamente os resultados. Mais especificamente, a inclusão da hipótese de endogeneidade da taxa natural de crescimento faz com que os principais resultados dessa abordagem sejam revertidos: que a moeda se torne não neutra no longo prazo e que os impactos de curto prazo passem a influenciar o desempenho da economia de longo prazo.

Porém, antes de analisar especificamente este ponto, cabe ressaltar que este debate, apesar de se apresentar como "novo", tem sua origem na síntese neoclássica proposta nos anos quarenta. Dessa síntese, o que deve ser destacado para a discussão aqui, em especial, é o consenso de que o equilíbrio com pleno emprego constitui o estado normal de uma economia de mercado, haja vista a existência de forças endógenas de convergência em direção à posição de pleno emprego dos fatores produtivos. Em outras palavras, a conclusão determinada pela síntese é de que o market clearing simultâneo em todos os mercados gera um processo de ajustamento contínuo (tâtonnement) que leva à configuração de um equilíbrio com pleno emprego. Além disso, ainda que temporariamente, admite-se que essas forças endógenas de convergência podem ser contrapostas pela existência de diversas formas de rigidez de preço e/ou salários e de outras imperfeições, podendo levar a situações de equilíbrio em que prevalece o excesso de oferta de trabalho no curto prazo e, consequentemente, ocorrendo este (equilíbrio) em situações pareto inferiores com produto abaixo do potencial.

Assim, como explicitado anteriormente, entende-se que esses aspectos teóricos constituem, em grande medida, o "plano de fundo" do Novo Consenso Macroeconômico, em que se acrescentam algumas sofisticações em termos da 
estrutura teórica e, principalmente, em termos formais. No entanto, as questões são as mesmas que permearam o debate da síntese neoclássica.

As críticas em relação a essa síntese são bastante conhecidas na literatura e não serão retomadas aqui. Todavia, um ponto essencial dessa discussão é a crença na existência de um equilíbrio de pleno emprego para o qual converge a economia no longo prazo. Como demonstrado por diversos trabalhos na literatura heterodoxa, como em Amadeo (1988), Oreiro (1997), Missio e Oreiro (2008), entre outros, essa conclusão é sujeita a diversas críticas e tende a ser viesada na medida em que desconsidera da análise importantes efeitos de uma deflação de preços sobre a demanda agregada, como proposto por Keynes no capítulo 18 da Teoria Geral.

Com relação a esse ponto, o trabalho de Missio e Oreiro (2008) mostra que, admitindo-se o mesmo conjunto de hipóteses que a síntese neoclássica, é possível, a partir da formalização dos argumentos de uma deflação de preços sobre a demanda agregada, evidenciar duas importantes implicações defendidas pela economia Keynesiana: i) de que a economia é inerentemente instável, sendo esta instabilidade limitada e ii) de que, como norma geral, é de se esperar que, no longo prazo, a economia opere com subutilização de recursos. Segundo Keynes:

An outstanding characteristic of the economic system in which we live that, whilst it is subject to severe fluctuations ... it is not violently unstable. Indeed it seems capable of remaining in a chronic condition of sub-normal activity for a considerable period (CWJMK, v. VII, 1973, p. 249).

A hipótese de trajetória única, ou de equilíbrio único, é veementemente criticada pela teoria pós-keynesiana. A partir das formalizações dos argumentos da Teoria Geral por diversos autores, entre eles, Kregel (1976), Dutt (1997) e Setterfield (1999), é possível mostrar que a trajetória de equilíbrio da economia passa a ser caracterizada pela presença de equilíbrios múltiplos, em que se destacam as dependências de trajetória, os processos de causalidade cumulativa e, sobretudo, o fim da dicotomia clássica entre o lado real e o lado monetário.

Esses resultados são derivados também a partir da análise da estacionariedade das séries macroeconômicas. Em primeiro lugar, vale ressaltar que a teoria macroeconômica tradicional supõe a existência de estacionariedade nas séries, e a análise passa a ser feita, então, com base na decomposição da hipótese ciclo-tendência. Mais especificamente, admite-se que flutuações cíclicas ocorrem no curto prazo, flutuando em torno de uma tendência sobre a qual a economia se desloca ao longo do tempo. Assim, à medida que os choques se dissipam, a influência do curto sobre o longo prazo torna-se nula. Ainda nesse contexto, destaca-se que as explicações mais convencionais para a existência dos ciclos são os choques monetários, enquanto os choques tecnológicos assumem o papel principal na explicação do componente tendencial. 
Em segundo lugar, em termos de análise das implicações de política econômica, isso significa que é possível separar o componente "cíclico" da "tendência" e, portanto, dentro desse arcabouço teórico, continuam "válidas" algumas hipóteses e resultados fundamentais como a neutralidade da moeda a longo prazo, a separação clássica entre o lado real e monetário da economia, a existência de um único e estável equilíbrio e o fato de o produto de longo prazo ser influenciado somente por choques de oferta, entre outros.

Todavia, a existência de raízes unitárias nas séries, em linha com o trabalho pioneiro de Nelson e Plosser (1982), caminha em sentido oposto à interpretação anterior, na medida em que a não estacionariedade é um argumento contrário à decomposição ciclo-tendência. Esse resultado, integrado à visão keynesiana e pós-keynesiana do comportamento dinâmico de uma economia capitalista de produção, mostra que a existência dessas características nas séries macroeconômicas faz com que choques a curto prazo (ciclo) causem movimentos persistentes no produto de longo prazo (tendência). Assim, as interpretações sobre as causas das flutuações e da dinâmica do produto ao longo do tempo mudam sensivelmente.

Nesse contexto, os choques de demanda passam a influenciar o produto e isso evidencia, entre outros fatores, que a moeda é não neutra (fim da dicotomia clássica); que a trajetória do produto depende do acúmulo de choques (de curto prazo) e que a variância da série cresce com o horizonte de previsão, o que significa que as estimativas para horizontes maiores de tempo ficam comprometidas. Portanto, as análises devem levar em consideração a existência de path dependence, de equilíbrio múltiplos, de hysteresis e de incerteza em relação ao comportamento futuro das variáveis, como preconizado por Dutt (1997), Dutt e Ros (2007), entre outros.

Por outro lado, devem também ser consideradas algumas inconsistências das distintas teorias que compõem o novo consenso. Nesse caso, observa-se que o Novo Consenso Macroeconômico incorpora muitos resultados da teoria Novo Keynesiana, sendo essa teoria sujeita a inúmeras críticas. Sendo assim, no que se segue, elencam-se algumas dessas críticas, muito embora possam ser estendidas para as demais escolas que formam o consenso.

Primeiramente, deve-se destacar que a macroeconomia Novo Keynesiana fundamenta-se na teoria neoclássica e que, portanto, se caracteriza pela presença de agentes econômicos racionais, como as famílias, que maximizam a utilidade para dada restrição orçamentária de forma que os preços relativos são proporcionais às utilidades marginais e às empresas, que maximizam o lucro, de forma que o resultado é expresso na igualdade entre a remuneração de cada fator produtivo com sua produtividade marginal. Assim, dadas as preferências, a 
tecnologia e a dotação de fatores, são estabelecidas as condições intertemporais de equilíbrio das variáveis reais. Soma-se a isso a interpretação de que todo excesso de oferta de moeda se traduz em excesso de demanda por bens e serviços, com consequente elevação de preços.

Em segundo lugar, conforme destacou Gontijo (2007), essas características são insuficientes para gerar explicações acerca da flutuação do produto de curto prazo, ou ainda, acerca dos efeitos macroeconômicos, inclusive monetários, provocados por mudanças de preços relativos. Nesse caso, os novos keynesianos apontam para a existência da rigidez de salários e preços e os custos associados às mudanças dos valores dessas variáveis são as principais razões pelas quais a economia se ajusta lentamente a "choques". Isso significa, também, admitir a eficácia da política monetária, pelo menos a curto prazo.

Existe, no entanto, uma série de incoerências e inconsistências nos modelos de inspiração novo keynesiana que padecem de uma melhor explicação. Nesse caso, podem-se destacar, entre outros, o seguinte conjunto de críticas, na ótica de Gontijo (2007):

i) A primeira crítica a esta teoria é justamente quanto à hipótese de rigidez de preço. No contexto da microeconomia neoclássica, a presença de rigidez de preços e salários implica admitir perdas para trabalhadores e empresários e, mercê dos esforços em justificar essas perdas a partir de fatos estilizados, os mesmos permanecem, em grande medida, como colocações "ad hoc" (p. 6).

ii) Em segundo lugar, parece contraditória a hipótese de que, no longo prazo, o nível de preços seja determinado pela oferta de moeda e o fato de o controle inflacionário se dar pelo manejo da taxa real de juros que, conforme dispõe a teoria da preferência intertemporal de Fisher, é a variável real que não deveria afetar a taxa de inflação, mas a relação entre consumo presente e consumo futuro. Na verdade, tampouco parece claro como o banco central determina a taxa de juros, visto que a teoria neoclássica concebe a curva LM como vertical no espaço taxa de juros vs. renda nominal, para não falar no abandono de qualquer relação de longo prazo entre a oferta de moeda e o nível de preços (p. 7).

iii) Em terceiro lugar, apesar do apelo teórico da curva de Phillips novo keynesiana, existem trabalhos que mostram que este modelo prevê que políticas anti-inflacionárias geram expansão econômica, sendo problemático explicar a inércia inflacionária por seu intermédio ou possível sustentar a taxa de desemprego permanentemente abaixo da "taxa de desemprego natural", o que viola a hipótese de que a economia tende para sua "taxa natural" e que prevalece a dicotomia neoclássica no longo prazo (p. 7-8).

Outras críticas pontuais têm sido apontadas por essa literatura sobre a estrutura teórica em que se baseia o novo consenso. Essas críticas se originam das 
hipóteses que são utilizadas nesse modelo, dentre as quais, segundo Arestis (2007), destacam-se: i) o fato de que o estoque de moeda é determinado por "resíduo" e de que esta não tem nenhum feedback com outras variáveis e ii) de que a taxa de juros é determinada pelo banco central e não pelo mercado. A primeira característica engloba o conceito de endogeneidade da moeda, ou seja, a moeda passa a ser originária do sistema bancário decorrente do processo de criação de empréstimos, sendo que o estoque de moeda é essencialmente determinado pela demanda. A hipótese de endogeneidade da moeda tem várias implicações para o papel da política econômica dentro da abordagem não convencional póskeynesiana, como pode ser observado na teoria da preferência pela liquidez dos bancos (Carvalho, 1993; Paula, 1999) ou nas teorias que remetem às fases de desenvolvimento desse sistema (Chick, 1986). Assim, a forma como se interpreta o papel da política monetária muda sensivelmente com relação à abordagem convencional, uma vez que, agora, a moeda - que é o ativo com o atributo de liquidez máxima - tem papel fundamental, na medida em que, por um lado, passa a ser desejada pelos agentes, dadas as incertezas não probabilísticas que caracterizam a "visão" de mundo dentro dessa abordagem e, por outro, porque o desejo por liquidez (moeda) afeta as decisões referentes à composição dos portfólios dos agentes, ou seja, a moeda torna-se não neutra.

Ainda, nesse contexto, deve-se observar que um dos pilares do novo consenso é a ideia de que a autoridade monetária deve ter como objetivo principal a estabilidade de preços, operacionalizada por um regime de metas de inflação e de um Banco Central Independente (BCI), cuja interpretação deriva da hipótese de que, no longo prazo, existe um único equilíbrio de pleno emprego e de que a moeda é neutra.

A crítica a essa interpretação, do ponto de vista da literatura póskeynesiana, advém do fato de que para esses autores, numa economia capitalista de produção, os capitalistas buscam preservar e aumentar sua riqueza pela posse de ativos diversos, como bens de capital, ativos financeiros, moeda, entre outros. Assim, neste tipo de sociedade, a política monetária tem o poder de alterar a rentabilidade dos diversos ativos de tal forma que, em determinado momento, os capitalistas podem preferir valorizar sua riqueza em ativos cuja ampliação não implique geração de emprego, como é o caso dos ativos financeiros. Ou seja, a política monetária pode permitir que a valorização do capital ocorra no chamado circuito financeiro e não no circuito produtivo, levando a economia a um equilíbrio de longo prazo com a presença de desemprego involuntário (Crocco; Jame Jr., 2003; Oreiro, 1999). Ou seja, a moeda é não neutra.

Por outro lado, deve-se considerar que, em grande parte, a teoria que fundamenta a preposição da necessidade de BCI e de outras propostas defendidas pelo novo consenso baseia-se principalmente nos argumentos propostos por 
Kydland-Prescott (1977). Entretanto, como mostrado por Sicsú (1997, p. 50) cabe perguntar a este modelo o seguinte: somente os policymakers desconhecem que não são capazes de surpreender os agentes e continuam inflacionando as economias sem saber que não conseguirão causar impactos nas variáveis reais? Os policymakers sabem que o público conhece o seu modelo de tomada de decisão? Se a resposta a esta última pergunta for negativa, o modelo de Kydland-Prescott estaria pressupondo que todos os agentes formam expectativas racionais, menos os policymakers! Se a resposta for positiva, o modelo careceria de lógica interna porque os policymakers estariam dispostos a realizar algo que saberiam ser impossível.

Do ponto de vista empírico, existe também uma série de críticas às proposições do novo consenso. Em primeiro lugar, não há evidência suficiente que sustente a argumentação de que a adoção de regimes de metas (de inflação) leve à redução da taxa de inflação. Mais especificamente, a relação de causalidade é, em grande medida, proposta como "ad hoc" (Kumhof, 2001; Sicsú, 2002; Arestis; Sawyer, 2003). O mesmo argumento vale em relação à hipótese de que bancos centrais independentes determinam uma menor taxa de inflação. Nesse caso, acrescentam-se as críticas em relação aos indicadores de independência que têm sido propostos até então (Carvalho, 1995-96; Forder, 1999; Mendonça, 2000, 2001, entre outros). Em segundo lugar, questiona-se a assimetria inerente à condução da política monetária em um regime de metas de inflação, ou seja, se essa política pode levar a um viés altista na taxa de juros, uma vez que, quando a inflação aumenta, a taxa de juros aumenta mais que proporcionalmente, sendo o inverso não verdadeiro (Libânio, 2009).

No mesmo sentido, seguem-se as críticas quanto à dificuldade de estimação e de ajustamento da regra de Taylor. Em primeiro lugar, porque ela pode ser escrita com base em variáveis difíceis de mensurar, como a taxa real de câmbio e/ou as expectativas de inflação e, em segundo lugar, porque em muitas estimativas evidencia-se seu "pobre" ajustamento.

Por fim, cumpre fazer alguns apontamentos que indicam quatro principais temas desenvolvidos pela literatura não convencional, sobretudo pós-keynesiana, que, como era de se esperar, são excluídos do novo consenso, já que o núcleo teórico dessas abordagens é distinto, e as mesmas, em grande medida, inconciliáveis, embora representem importantes contribuições desse pensamento no sentido de gerar novas interpretações acerca do entendimento de como ocorre o processo de crescimento (flutuações) do produto nas economias capitalistas de produção. 
O primeiro desenvolvimento refere-se ao conceito de endogeneidade da taxa natural de crescimento ${ }^{7}$. Por um lado, como mostrado por Lavoie (2007), essa hipótese, quando inserida no arcabouço teórico do novo consenso, muda radicalmente suas conclusões. Por outro, chama-se a atenção para o fato de que, do ponto de vista teórico e empírico, há uma série de argumentos e evidências que sustentam essa hipótese (Leon-Ledesma, 2002; Thirlwall, 2005; Libânio, 2009).

O segundo ponto refere-se à insuficiência da teoria neoclássica do crescimento e aos processos de causalidade cumulativa. A abordagem, aqui, destaca as limitações dos modelos de crescimento mainstream, tanto do ponto de vista teórico quanto do seu ajustamento à evidência empírica (Possas, 1999), o que leva à retomada da abordagem heterodoxa quanto à interpretação dos mecanismos que explicam o crescimento, em especial, a partir da análise de fatores condicionantes da demanda. Dentre esses, destacam-se as abordagens que possuem origem nas interpretações de Kaldor, sobretudo, nos processos de retroalimentação e de causalidade cumulativa (Dixon; Thirlwall, 1975; Thirlwall, 1979).

O terceiro refere-se aos modelos de crescimento com restrição no Balanço de Pagamentos (BP). Essa abordagem defende que as restrições da BP podem influenciar a taxa de crescimento da economia na medida em que ela pode determinar a subutilização da capacidade dos recursos existentes. Ou seja, é possível que, antes que as restrições de oferta comecem a operar, as condições de restrição da demanda assim o façam, determinando que a economia opere sempre abaixo do ponto de pleno emprego, ou seja, determinando uma taxa de crescimento menor que a do produto potencial. Assim, em termos de política econômica, o importante é saber quais os determinantes dessa taxa de crescimento e, mais importante, como é possível relaxar esta restrição. Nesse caso, os modelos de crescimento com restrição no balanço de pagamentos fornecem a base teórica para essa questão, como pode ser observado no trabalho seminal de Harrod (1939), seguido por Kaldor (1957) e Thirlwall (1979), entre outros.

Por fim, o último desenvolvimento refere-se ao papel da moeda e dos bancos na economia. Nessa abordagem, um dos principais resultados derivados da teoria keynesiana da escolha de portfólio é que variações no estoque de moeda são capazes de influenciar o estoque de capital de equilíbrio de longo prazo na economia. A razão apresentada como explicação é que, num ambiente caracterizado pela incerteza (tipo Knight - Keynes), a moeda passa a ser demandada como um ativo concorrente com outros ativos, entre eles, o capital, pela preferência dos agentes no processo de determinação da composição dos seus

(7) A taxa natural de crescimento é tradicionalmente definida como a soma da taxa de crescimento da força de trabalho e da taxa de crescimento da produtividade. Nesse caso, esse conceito de endogeneidade da taxa de crescimento admite que essas taxas são influenciadas pelo comportamento da demanda. 
portfólios. Nesse caso, a ideia é de que os agentes podem valorizar a posse de ativos líquidos, atribuindo um "prêmio de liquidez" à moeda. Ou seja, a moeda passa a apresentar um retorno implícito que concorre com o retorno explícito proporcionado pelos ativos de capital. Nesse sentido, um aumento da quantidade de moeda existente na economia pode fazer com que os agentes econômicos valorizem menos a posse de ativos líquidos, deslocando sua demanda em direção a outros ativos, em particular, os ativos de capital. Segue-se, portanto, que a não neutralidade da moeda é o resultado da preferência pela liquidez (Oreiro, 1999).

Além da não neutralidade da moeda, a literatura pós-keynesiana amplia também a interpretação referente ao papel do sistema financeiro, mais especificamente dos bancos comerciais. Nesse caso, as análises abordam o conceito de preferência pela liquidez desenvolvido por Keynes, que posteriormente foi ampliado e formalizado pelos pós-keynesianos, especialmente no que se refere à preferência pela liquidez do sistema bancário.

Nesse sentido, segundo Paula (1999, p. 03), os bancos são vistos como agentes ativos que administram dinamicamente os dois lados de seus balanços. Do ponto de vista do seu passivo, significa que eles não o tomam como dado, na medida em que procuram influenciar as preferências dos depositantes pelo gerenciamento das obrigações e da introdução de inovações financeiras. Ou seja, os bancos são vistos como entidades capazes de criar crédito independentemente da existência de depósitos prévios pela criação ativa da moeda bancária. Nesse caso, o comportamento dos bancos tem papel essencial na determinação das condições de financiamento, pois deles depende a criação de novo poder de compra necessário à aquisição de ativos de capital, que proporcionam a independência da acumulação de capital em relação à poupança prévia. Assim, como uma firma que possui expectativas e motivações próprias, seu comportamento tem impacto decisivo sobre as condições de financiamento da economia e, consequentemente, sobre o nível de gastos dos agentes, afetando as variáveis reais da economia, como produto e emprego.

\section{Considerações finais}

O objetivo desse trabalho consiste em fazer uma revisão da literatura acerca do Novo Consenso Macroeconômico por meio da análise dos principais autores que sustentam essa tese, bem como apresentar algumas considerações críticas apontadas pela literatura heterodoxa, sobretudo aquelas advindas da inspiração pós-keynesiana.

Inicialmente foi apresentada uma síntese da proposta do Novo Consenso, destacando-se seus objetivos e características. Nesse caso, destacam-se as proposições quanto à condução da política monetária via manipulação da taxa de 
juros e, principalmente, a concepção de que o objetivo central da autoridade monetária deve ser o de manter a estabilidade do nível de preços. Foi apresentada também a estrutura formal comum dos modelos que compõem essa abordagem teórica, com destaque para as três equações principais: a "nova" curva IS, uma curva de Phillips e uma Regra de Conduta semelhante à Regra de Taylor.

Do ponto de vista prático, os adeptos desse Novo Consenso defendem que o produto desse debate teórico pode ser encontrado nos resultados "positivos" dessa nova forma de condução da política monetária, uma vez que esta política tem sido adotada por bancos centrais de diversos países, como Canadá, Austrália, Inglaterra, Brasil, Chile, Colômbia, Israel, México, Peru, Suécia, Suíça, Turquia, Tailândia, Banco Central Europeu e Federal Reserve dos Estados Unidos. Particularmente, defende-se que o Brasil tenha se enquadrado nesse novo consenso, a partir de junho de 1999, com a desvalorização cambial e a adoção de um novo regime monetário à luz do regime de metas de inflação.

Por outro lado, a crítica heterodoxa - sobretudo a pós-keynesiana - baseiase na interpretação de que a trajetória de crescimento da economia é caracterizada por processos que exibem path dependence, equilíbrios múltiplos e hysteresis e, portanto, mostra-se incompatível com a interpretação proposta pelo Novo Consenso.

Em outras palavras, do ponto de vista da heterodoxia, a proposta de um novo consenso macroeconômico - que em sua essência segue a "velha" interpretação da síntese neoclássica de existência de uma trajetória de equilíbrio de longo prazo sobre o qual a economia se desloca ao longo do tempo, somado a novas interpretações quanto à forma e à influência das políticas econômicas, em especial, da política monetária - padece de inconsistências teóricas e é, em grande medida, a reprodução das questionáveis conclusões da síntese neoclássica dos anos quarenta.

Além disso, esse novo consenso, como proposto, de "síntese" do debate que caracteriza a evolução da macroeconomia recente é, essencialmente, um consenso mainstream. Sendo assim, na medida em que este mesmo consenso não incorpora os desenvolvimentos da teoria heterodoxa, ou, ainda, na medida em que este deriva de uma interpretação reducionista dos aspectos econômicos, ele não pode ser interpretado como consenso em termos de teoria econômica - a não ser no ambiente restrito em que está inserido.

Por fim, a principal conclusão desse trabalho aponta no sentido de que não existe a possibilidade, a julgar pelas evidências existentes, de que seja possível determinar um consenso entre essas duas abordagens ou determinar, como propõem os defensores desse Novo Consenso, um pensée unique em economia. 


\section{Referências bibliográficas}

AMADEO, E. As diferentes facetas da rigidez e flexibilidade dos salários na análise Keynesiana. Revista de Economia Política, v. 8, n. 1, jan./mar. 1988.

ANGERIZ, A.; ARESTIS, P. Monetary policy in the UK. Cambridge Journal of Economics, p. 1-22, Aug. 2007.

ARESTIS, P. What is the new consensus in macroeconomics. In: ARESTIS, Philip. Is there a new consensus in macroeconomics? New York: Palgrave Macmillan, 2007.

; SAWYER, M. New consensus macroeconomics and inflation targeting: Keynesian critique. Economia e Sociedade, Campinas, v. 17, Número especial, Dec. 2008.

. Can monetary policy affect the real economy? Annandale-on-Hudson, New York: The Levy Economics Institute, 2002a. (Working Paper Series, n. 355).

. Does the stock of money have any causal significance? Annandale-on-Hudson, New York: The Levy Economics Institute, 2002b. (Working Paper Series, n. 363).

. New Keynesianism and the economics of the "third way". Annandale-onHudson, New York: The Levy Economics Institute, 2002c. (Working Paper Series, n. 364).

. Inflation targeting: a critical appraisal. Levy Economics Institute of Bard College, Sept. 2003. (Working paper, n. 388).

The nature and role of monetary policy when money is endogenous. Cambridge Journal of Economics, v. 30, p. 847-860, Aug. 2006.

BARRO, R.; GORDON, D. A positive theory of monetary policy in a natural rate model. Journal of Political Economy, v. 91, n. 4, p. 589-610, Aug. 1983a.

; GORDON, D. Rules, discretion and reputation in a model of monetary policy. Cambridge: National Bureau of Economic Research, Feb. 1983b. (NBER Working Paper, n. 1079).

BEAN, C. Is there a new consensus in monetary policy (2007) In: ARESTIS, Philip. Is there a new consensus in macroeconomics? New York: Palgrave Macmillan, 2007.

BLANCHARD, O. J. Is there a core of usable macroeconomics? The American Economic Review, v. 87, n. 2, p. 244-246, May 1997.

BLINDER, A S. Is there a core of practical macroeconomics that we should all believe? The American Economic Review, v. 87, n. 2, p. 240-243, May 1997.

CALVO, G. Staggered prices in a utility-maximizing framework. Journal of Monetary Economics, v. 12, p. 383-398, 1983.

CARVALHO, F. C. Sobre a endogenia da oferta de moeda: réplica ao professor Nogueira da Costa. Revista de Economia Política, v. 13, n. 3 (51), jul./set. 1993.

. The independence of Central Banks: a critical assessment of the arguments. Journal of Post Keynesian Economics, v. 18, n. 2, Winter 1995-96.

CLARIDA, R.; GALÍ, J.; GERTLER, M. Monetary policy rules in practice: some international evidence. European Economic Review, n. 42, p. 1033-1067, 1998. 
CLARIDA, R.; GALÍ, J.; GERTLER, M. The science of monetary policy: a new Keynesian perspective. Cambridge: National Bureau of Economic Research, May 1999. (NBER Working Paper, n. 7147).

; _ $\quad$. Monetary policy rules and macroeconomic stability: Evidence and some theory. The Quarterly Journal of Economics, Feb. 2000.

CHICK, Victoria. A evolução do sistema bancário e a teoria da poupança, do investimento e dos juros. Ensaios FEE, Porto Alegre, v. 15, n. 1, p. 9-23, 1994 (Edição original: 1986).

CROCCO, M.; JAYME JR., F. Independência e autonomia do Banco Central: mais sobre o debate. Belo Horizonte, Cedeplar, 2003. (Texto para Discussão, n. 199).

DIXON, R.; THIRLWALL, A. (1975). A model of regional growth-rate differences on Kaldorian lines. In: KING, J. E. Economic growth in theory and practice. Aldershot: Edward Elgar, 1994. (The International Library of Critical Writings in Economics series, n. 43).

DUTT, A. Equilibrium, path dependence and hysteresis in post-Keynesian models. In: ARESTIS, Philip; PALMA, Gabriel; SAWYER, M. (Ed.). Markets, unemployment and economic policy - essays in Honor of Geoff Harcourt. New York: Routledge, 1997. v. 2.

; ROS, J. Aggregate demand shocks and economic growth. Structural Change and Economic Dynamics, v. 18, issue 1, 2007.

FORDER, J. Central bank independence: reassessing the measurements. Journal of Economic Issues, v. XXXIII, n. 1, Mar. 1999.

FISCHER, S. Long-term contracts rational expectations and the optimal money supply rule. Journal of Political Economy, v. 85, n. 1, p. 191-205, 1977.

GALÍ, J. Monetary policy, inflation, and the business cycle. An introduction to the new Keynesian framework. Princeton University, 2008.

GOODFRIEND, M.; KING, R. G. The new neoclassical synthesis and the role of monetary policy. In: BERNANKE, B.; ROTEMBERG, J. NBER Macroeconomics Annual. The MIT Press, Jun. 1997.

. The monetary policy debate since october 1979: lessons for theory and practice. Federal Reserve Bank of St. Louis Review, 2, p. 243-262, Mar./Apr. 2005.

How the world achieved consensus on monetary policy. Cambridge: National Bureau of Economic Research, Nov. 2007. (NBER Working Paper, n. 13580).

GONTIJO, C. Notas críticas sobre a macroeconomia novo-keynesiana. Belo Horizonte: Cedeplar, 2007. (Textos para Discussão, n. 320).

JUDD, J.; RUDEBUSH, G. D. Taylor's rule and the Fed: 1970-1997. Federal Reserve Bank of San Francisco. Economic Review, n. 3, p. 3-16, 1998.

HARROD, R. An essay in dynamic theory. Economic Journal, v. 49, p. 14-33, 1939.

KALDOR, N. A model of economic growth. The Economic Journal, v. 67, n. 268, p. 591$624,1957$.

KEYNES, J. M. The collected writings of John Maynard Keynes. Editado por D. E. Moggridge. London: Macmillan, 1973. [no texto é referenciado por CWJMK]. 
KREGEL, J. Economic methodology in face of uncertainty: the modeling methods of Keynes and post- Keynesians. Economic Journal, v. 86, n. 342, 1976.

KUMHOF, M. A critical view of inflation targeting: crises, limited sustainability and aggregate shocks. Central Bank of Chile, 2001. (Working Papers, n. 127).

KYDLAND, F.; PRESCOTT, E. (1977). Rules rather than discretion: the inconsistency of optimal plans. In: LUCAS, R.; SARGENT, T. Rational expectations and econometric practice. Minnesota: The University of Minnesota Press, 1984. p. 229-260.

LAVOIE, M. A post Keynesian amendment to the new consensus on monetary policy. Metroeconomica, v. 57, n. 2, p. 165-192, 2006.

LIBÂNIO, G. A. Aggregate demand and the endogeneity of the natural rate of growth: evidence from Latin American economies. Cambridge Journal of Economics, v. 33, p. 1, 2009.

- A note on inflation targeting and economic growth in Brazil. Revista de Economia Política, 2009 (a ser publicado).

MANKIW, N. G. The inexorable and Mysterious tradeoff between inflation and unemployment. Economic Journal, May 2001.

McCALLUM, B. T. Recent developments in monetary policy analysis: the roles of theory and evidence. Cambridge: National Bureau of Economic Research, Apr. 1999. (NBER Working Paper, n. 7088).

- Monetary policy analysis in models without money. Federal Reserve Bank of St. Louis Review, Jul./Aug. 2001.

. What have learned since October 1979? Federal Reserve Bank of St. Louis Review, Mar./Apr. part 2, 2005.

MEYER, L. H. Does money matter? Federal Reserve Bank of St. Louis Review, p. 1-15, 2001.

MENDONÇA, H. F. A teoria da independência do Banco Central: uma interpretação crítica. Estudos Econômicos, Instituto de Pesquisas Econômicas - USP, São Paulo, v. 30, n. 1, 101-127, 2000.

. A mensuração do grau de independência do Banco Central: uma análise de suas fragilidades. Análise Econômica, Faculdade de Ciências Econômicas, UFRGS, Ano19, n. 36, p. 47-67, set. 2001.

MISHKIN, F. Will monetary become more of a science? Cambridge: National Bureau of Economic Research, Oct. 2007. (NBER Working Paper, n. 13566).

MISSIO, F.; OREIRO, J. L. Equilíbrio com desemprego involuntário em um modelo de ciclo-limite. Revista Economia, 2009 (a ser publicado).

NELSON, C.; PLOSSER, C. Trends and random walks in macroeconomics time series, some evidence and implications. Journal of Monetary Economics, 10, p. 139-162, 1982.

OREIRO, J. L. Flexibilidade salarial, equilíbrio com desemprego e desemprego de desequilíbrio. Revista Brasileira de Economia, v. 51, 1997. 
OREIRO, J. L. Preferência pela liquidez e escolha de portfólio: estabelecendo os microfundamentos da não-neutralidade no longo prazo. Análise Econômica, UFRGS, v. 17, n. 32, p. 87-108, 1999.

PAULA, L. F. Dinâmica da firma bancária: uma abordagem não-convencional. Revista Brasileira de Economia, v. 53, n. 3, p. 323-356, 1999.

POSSAS, M. Antecedentes e perspectivas teóricas da economia do desenvolvimento numa abordagem evolucionária. Nexos Econômicos, Salvador, v. 1, n. 1, 1999.

SETTERFIELD, M. Expectations, path dependence and effective demand: a macroeconomic model along Keynesian lines. Journal of post Keynesian Economics, v. 21, n. 3, 479-501, Spring 1999.

SICSÚ, J. A negação da ineficácia da política monetária: a alternativa de Keynes e dos pós-keynesianos. Análise Econômica, v. 15, n. 28, p. 80-107, 1997.

- Teoria e evidências do regime de metas inflacionárias. Revista de Economia Política, v. 22, n. 1 (85), jan./mar. 2002.

TAYLOR, J. Staggered wage setting in a Macro Model. American Economic Review, v. 69. n. 2, p. $108-113,1979$.

. A core of practical macroeconomics. The American Economic Review, v. 87, n. 2, p. 233-235, May 1997.

. Teaching modern macroeconomics at the principles level. American Economic Review, v. 90, n. 2, May 2000.

THIRLWALL, A. P. A endogeneidade da taxa natural de crescimento. In: A NATUREZA do crescimento econômico: um referencial alternativo para compreender o desempenho das nações. Brasília, IPEA, 2005.

. The balance of payments constraint as an explanation of international growth rate differences. Banca Nazionale del Lavoro Quarterly Review, n. 128, Mar. 1979.

WOODFORD, M. Revolution and evolution in twentieth century macroeconomics. Princeton University, Jun. 1999.

. Convergence in macroeconomics: elements of the new synthesis. Columbia University, Jan. 2008.

. Interest and prices: foundations of a theory of monetary policy. Princeton University Press, 2003.

ZOUACHE, A. Towards a new neoclassical synthesis? An analysis of the methodological convergence between new Keynesian economics and real business cycle theory. History of Economic Ideas, XII, 2004. 